\title{
Monogenic lupus due to spondyloenchondrodysplasia with spastic paraparesis and intracranial calcification: case-based review
}

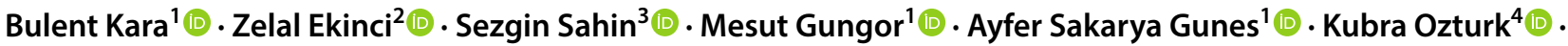

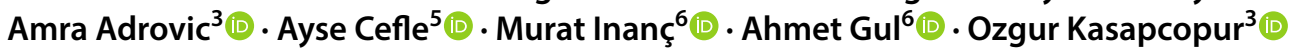

Received: 12 May 2020 / Accepted: 11 July 2020 / Published online: 20 July 2020

(c) Springer-Verlag GmbH Germany, part of Springer Nature 2020

\begin{abstract}
Spondyloenchondrodysplasia (SPENCD) is a rare skeletal dysplasia characterized with platyspondyly and metaphyseal lesions of the long bones mimicking enchondromatosis, resulting in short stature. SPENCD often coexists with neurologic disorders and immune dysregulation. Spasticity, developmental delay and intracranial calcification are main neurologic abnormalities. Large spectrum of immunologic abnormalities may be seen in SPENCD, including immune deficiencies and autoimmune disorders with autoimmune thrombocytopenia and systemic lupus erythematosus as the most common phenotypes. SPENCD is caused by loss of tartrate-resistant acid phosphatase (TRAP) activity, due to homozygous mutations in $A C P 5$, playing a role in non-nucleic acid-related stimulation/regulation of the type I interferon pathway. We present two siblings, 13-year-old girl and 25-year-old boy with SPENCD, from consanguineous parents. Both patients had short stature, platyspondyly, metaphyseal changes, spastic paraparesis, mild intellectual disability, and juvenile-onset SLE. The age at disease-onset was 2 years for girl and 19 years for boy. Both had skin and mucosa involvement. The age at diagnosis of SLE was 4 years for girl, and 19 years for boy. The clinical diagnosis of SPENCD was confirmed by sequencing of ACP5 gene, which revealed a homozygous c.155A > C (p.K52T), a variant reported before as pathogenic. Juvenile-onset SLE accounts for about 15-20\% of all SLE cases. But, the onset of SLE before 5-years of age and also monogenic SLE are rare. Our case report and the literature review show the importance of multisystemic evaluation in the diagnosis of SPENCD and to remind the necessity of investigating the monogenic etiology in early-onset and familial SLE cases.
\end{abstract}

Keywords SPENCDI $\cdot$ Spondyloenchondrodysplasia $\cdot A C P 5 \cdot$ Skeletal dysplasia $\cdot$ Systemic lupus erythematosus $\cdot$ Type I interferonopathy $\cdot$ Immune dysregulation

Ozgur Kasapcopur

ozgurkasapcopur@hotmail.com

Bulent Kara

bkuskudar@gmail.com

Zelal Ekinci

zekinci@outlook.com

Sezgin Sahin

sezgin@istanbul.edu.tr

Mesut Gungor

drmesutgungor@gmail.com

Ayfer Sakarya Gunes

ayfer-sakarya@hotmail.com

Kubra Ozturk

ozturk1209@gmail.com

Amra Adrovic

aadrovic@yahoo.com

Ayse Cefle

acefle@hotmail.com
Murat Inanç

minanc2008@gmail.com

Ahmet Gul

agul@istanbul.edu.tr

1 Department of Pediatric Neurology, Medical Faculty, Kocaeli University, Kocaeli, Turkey

2 Department of Pediatric Nephrology and Rheumatology, Florence Nightingale Hospital, Istanbul, Turkey

3 Department of Pediatric Rheumatology, Cerrahpasa Medical Faculty, Istanbul University-Cerrahpasa, Istanbul, Turkey

4 Department of Pediatric Rheumatology, Medical Faculty, Medeniyet University, Istanbul, Turkey

5 Department of Rheumatology, Medical Faculty, Kocaeli University, Kocaeli, Turkey

6 Department of Rheumatology, Istanbul Medical Faculty, Istanbul University, Istanbul, Turkey 


\section{Introduction}

Spondyloenchondrodysplasia (SPENCD) was first defined by Schorr et al. in two brothers with spondylometaphyseal dysplasia and enchondromatous changes from consanguineous Iraqi Jewish parents in 1976 [1]. In the following two to three decades, a few case reports indicated that some of SPENCD cases had intracranial calcifications and spastic paraparesis without explaining the association of skeletal and neurological findings [2-4]. In 2003, Roifman and Melamed described a novel syndrome of spondylometaphyseal dysplasia, combined immunodeficiency and autoimmunity in four patients, who had similar clinical and immunologic features suggesting the presence of immune dysregulation [5]. A pathogenetic relationship between spondyloenchondrodysplasia, spasticity, intracranial calcification, and immune dysregulation was first described by Renella et al. in 2006 [6]. They reported that the correlation between the severity of radiologic findings and the neurological impairment or immune dysregulation was not proportionate and thought that the responsible gene showed marked pleiotropism. Simultaneously, Briggs et al. and Lausch et al. identified homozygous or compound heterozygous mutations in the ACP5 gene, encoding tartrate-resistant acid phosphatase (TRAP), in patients with SPENCD, respectively from 10 patients in 8 families and 14 patients in 11 families [7,8]. The molecular basis of SPENCD was proposed as the loss of regulation of TRAP activity on osteopontin function (OPN) in both studies. The functional excess of phosphorylated OPN are thought to cause increased bone resorption by activated osteoclasts and immune dysregulation by stimulation of type I interferon production in plasmacytoid dendritic cells. Prior studies which showed elevated plasma OPN levels correlated with disease activity in systemic lupus erythematosus (SLE), and increased SLE susceptibility due to SNPs within the $S P P 1$ gene encoding OPN supported the findings related to immune dysregulation $[9,10]$. Briggs et al. found elevated serum type I interferon activity in all SPENCD subjects sampled as in Aicardi-Goutières syndrome, which is a type I interferonopathy with similar symptoms in SPENCD, such as intracranial calcification and susceptibility to SLE. In 2011, Crow identified SPENCD with Aicardi-Goutières syndrome and systemic lupus erythematosus with complement deficiency as type I interferonopathies [11].

SPENCD has been associated with a wide-spectrum of autoimmune diseases, such as systemic lupus erythematosus (SLE), Sjogren's syndrome, hemolytic anemia, thrombocytopenia, hypothyroidism, inflammatory myositis, Raynaud's disease and vitiligo, isolated or in combination [7]. In the comprehensive survey of Briggs et al.
$85 \%(22 / 26)$ of the patients had at least one autoimmune disease, and autoimmune thrombocytopenia (AITP) was the most common diagnosis [12]. SPENCD and SPENCD with immune dysregulation (SPENCDI) had been found as a continuum of the same disorder according to the molecular evidence by the same authors, and they proposed to use SPENCD for both phenotypes [12].

Tartrate-resistant acid phosphatase (TRAP) deficiency in SPENCD predisposes patients to develop SLE, mostly starting during childhood $[12,13]$. The probability of a monogenic etiology increases with the decreasing age of onset in SLE [14], and SPENCD is a one of the rare causes of monogenic forms of SLE. Here, we present two siblings with a diagnosis of SPENCD, who had juvenile-onset SLE. We also aim to discuss the importance of multisystemic evaluation in the diagnosis of SPENCD and to remind the importance of investigating the monogenic etiology in early-onset and familial SLE cases.

\section{Case report}

A 13-year old girl and a 25-year-old boy from a Turkish family were presented with similar clinical findings at the ages of 2 years and 19 years, respectively. The girl was third sibling of parents, who were second cousins. She was first hospitalized at 2 years of age due to respiratory distress, itching, erythematous rash on trunk and high fever. She had symmetric polyarthralgia involving large and small joints, cervical and axillary lymphadenopathy, and hepato-splenomegaly. Laboratory examination showed pancytopenia (leukocyte $2.270 / \mathrm{mm}^{3}$, hemoglobin $5.8 \mathrm{~g} / \mathrm{dl}$, thrombocyte $\left.33.900 / \mathrm{mm}^{3}\right)$, low erythrocyte sedimentation rate $(5 \mathrm{~mm} /$ hour), hypertriglyceridemia $(252 \mathrm{mg} / \mathrm{dL})$, elevated transaminase levels (AST 159 IU/L, ALT 51 IU/L), hyperferritinemia $(4.390 \mathrm{ng} / \mathrm{ml})$, and increased CRP levels $(4 \mathrm{mg} / \mathrm{dL})$. Bone marrow aspiration revealed hemophagocytosis. She was transferred to the intensive care unit and connected to mechanic ventilator for 2 days with a presumed diagnosis of macrophage activation syndrome secondary to systemic juvenile idiopathic arthritis. Computerized tomography of the chest revealed bilateral parenchymal pulmonary dense infiltrates and mild pleural effusion. After 3 days of intravenous pulse methylprednisolone $(30 \mathrm{mg} / \mathrm{kg} / \mathrm{day})$, treatment was continued with oral methylprednisolone $(2 \mathrm{mg} / \mathrm{kg} / \mathrm{day})$, methotrexate $\left(15 \mathrm{mg} / \mathrm{m}^{2} /\right.$ week $)$ and cyclosporine $(3 \mathrm{mg} / \mathrm{kg} /$ day). She improved significantly in a few weeks. Corticosteroid, cyclosporine and methotrexate were discontinued after 1,3 and 14 months of therapy, respectively. At the age of 3.5 years, she had a direct Coombs positive hemolytic anemia episode, and after a short time presented with walking disability due to generalized joint pain and fever, both of which improved with pulse methylprednisolone $(30 \mathrm{mg} / \mathrm{kg} /$ day) for 3 days. Prolonged PTT levels and leukopenia were 
noticed during these episodes. At the age of 4 years, when she was not on any immunosuppressants, she presented again with walking disability, symmetric polyarthralgia, oral aphthous ulcers, malar rash and photosensitivity. Short stature was noted for the first time. Exaggerated deep tendon reflexes, clonus and paraparesis, all of which are suggestive of an upper motor neuron lesion, were detected on examination. Laboratory investigations showed low C3 $(41.6 \mathrm{mg} / \mathrm{dl}$; normal range $90-180)$ and $\mathrm{C} 4$ levels $(7.09 \mathrm{mg} / \mathrm{dl}$; normal range 10-40).

Antinuclear antibodies (ANA) were strongly positive with homogeneous pattern, and anti-double-stranded DNA antibodies were detected at borderline level (25.7 IU/L, normal $<24 \mathrm{IU} / \mathrm{L}$ ). She was diagnosed with systemic lupus erythematosus, and after a 3-day course of pulse methylprednisolone (30 mg/kg/day), oral methylprednisolone ( $2 \mathrm{mg} / \mathrm{kg} /$ day $)$, azathioprine $(2 \mathrm{mg} / \mathrm{kg} /$ day $)$ and hydroxychloroquine $(5 \mathrm{mg} / \mathrm{kg} /$ day) were initiated. Leukopenia persisted despite these drugs. Endocrinological investigations for short stature were unremarkable. Skeletal radiographies showed platyspondyly and metaphyseal hyperdense lesions (Fig. 1d, e). Brain magnetic resonance imaging (MRI) showed mild ventricular dilatation due mild cerebral atrophy, without signal abnormality (Fig. 1c). At the age of 7 years she had pharyngeal petechiae, and complete blood count showed bicytopenia with a platelet count of $2.000 / \mathrm{mm}^{3}$ and leukocyte count of $2.500 / \mathrm{mm}^{3}$ (neutrophils $1.600 / \mathrm{mm}^{3}$, and lymphocytes $900 / \mathrm{mm}^{3}$ ). Bicytopenia was improved after a single dose of intravenous immunoglobulin treatment $(1 \mathrm{gr} / \mathrm{kg})$ and pulse methylprednisolone (30 mg/kg/day), for 5 days. At follow-up, she had no other deterioration.

She was born at term, 3.100 g. Prenatal, natal, and postnatal periods were uneventful. She sat at the age of 8 months, walked and spoke single words at 18 months. Parents noticed that her face looked red since she was born, and wounds appeared in the itchy areas. The parents were consanguineous, and had 4 siblings (one boy, three girls).
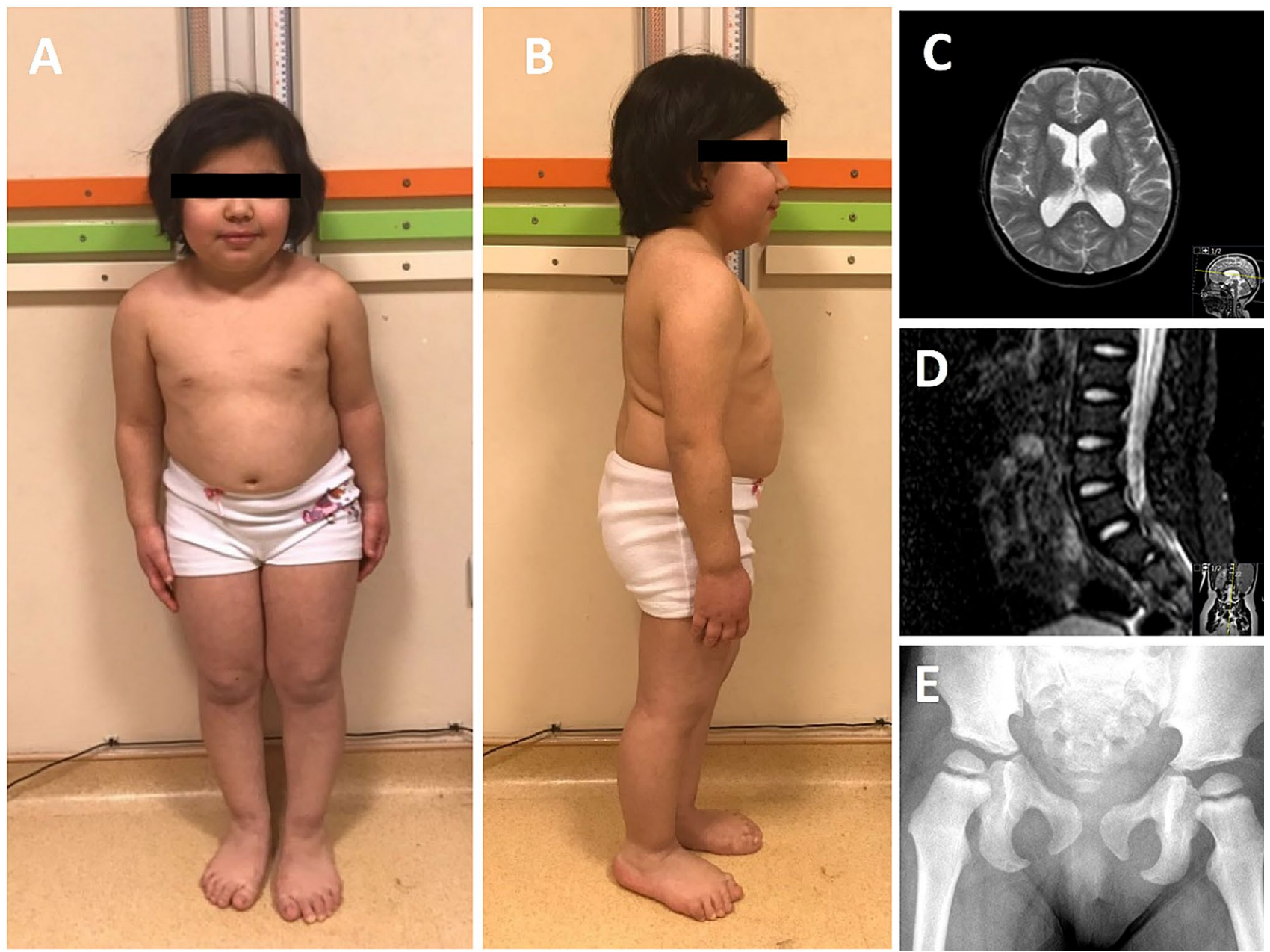

Fig. 1 Photographs of the proband (a, b). Magnetic resonance imaging of the brain shows mild ventricular dilatation and cortical atrophy at T2-weighted axial image (c). Platyspondyly of the lumbar vertebrae (d). Metaphyseal hyperintensity of proximal femur (e) 
At the last physical examination, her weight was $25 \mathrm{~kg}$ $(<3 p ;-4,56 \mathrm{SD})$, height $106 \mathrm{~cm}(<3 p ;-8,64 \mathrm{SD})$, and head circumference $51 \mathrm{~cm}(<3 p ;-2,71 \mathrm{SD})$ (Fig. 1a, b). She had mild cognitive impairment and upper motor neuron signs at lower extremities (increased deep tendon reflexes, Achille's clonus and positive Babinski reflexes). She had no organomegaly and lymphadenopathy. At present, she is 13 -year old, and using azathioprine $(2 \mathrm{mg} / \mathrm{kg} /$ day) and hydroxychloroquine ( $4 \mathrm{mg} / \mathrm{kg} /$ day). Laboratory investigations at last visit showed low leukocyte and lymphocyte counts and presence of anti-ds DNA antibodies in her serum at high levels.

The brother of the index case was first reported to the hospital at 19 years of age because of recent onset skin rash prominent on feet and legs, oral aphthous ulcers, skin sores, and joint stiffness in the fingers, knees and elbows in the morning. He had no history of arthritis, malar rash, and photosensitivity. He had significant proteinuria, and kidney biopsy showed histopathologic findings of class II lupus nephritis. His ANA was positive with homogeneous pattern, and ENA investigation revealed high titer antibodies against $\mathrm{Sm}$ and $\mathrm{Sm} / \mathrm{RNP}$. He also had anti-dsDNA antibodies, and both $\mathrm{C} 3$ and $\mathrm{C} 4$ complement levels were low (44 and $6.1 \mathrm{mg} / \mathrm{dl}$, respectively). Prednisolone $(60 \mathrm{mg} /$ day), hydroxychloroquine (200 $\mathrm{mg} /$ day), and azathioprine $(100 \mathrm{mg} /$ day $)$ were started, and he responded well to the treatment.

One year after the diagnosis, he presented with abdominal pain, vomiting and nausea. Abdominal computed tomography revealed edema and inflammation of jejunum and duodenum. The corticosteroid dosage has been increased (60 mg/day). After a few months, the symptoms recurred. His anti-phospholipid antibodies were negative, and no findings of venous or arterial thrombosis was found during the follow-up. The patient was thought to have gastrointestinal involvement due to SLE. Addition of rituximab treatment $\left(375 \mathrm{mg} / \mathrm{m}^{2}\right.$ IV once weekly $\times 4$ doses) after the last episode provided a clinical remission, and no other deterioration related to acute gastrointestinal system has been experienced thereafter.

One year after the diagnosis of SLE, he had recurrent episodes of severe nausea and abdominal pain, and partial thrombosis was detected in the main and right portal vein. Low molecular weight heparin was added to his treatment for 6 months, and he then continued to receive $100 \mathrm{mg}$ acetyl salicylic acid. His anti-phospholipid antibodies were negative, and no findings of venous or arterial thrombosis was found during the follow-up. Laparoscopic investigation due to episodes of abdominal pain revealed no significant pathological finding, and his complaints were controlled with high dose corticosteroids ( $60 \mathrm{mg} /$ day). His complaints were thought to be associated with the disease activity, addition of rituximab treatment $\left(375 \mathrm{mg} / \mathrm{m}^{2} \mathrm{IV}\right.$ once weekly $\times 4$ doses) after the last episode provided a clinical remission, and no other deterioration has been experienced until now.

He was born at term, 3.050 g. Prenatal, natal, and postnatal periods were uneventful. He walked and spoke single words at 14 months. He had a history of frequent falls for the past 5 years. He was diagnosed as spastic paraparesis, but how long this clinical finding was unknown.

At last physical examination, his weight was $58 \mathrm{~kg} \mathrm{(6p}$; $-1,61 \mathrm{SD})$, height $144 \mathrm{~cm}(<3 p ;-5,22 \mathrm{SD})$, and head circumference $56,1 \mathrm{~cm}(15 p ;-1,07 \mathrm{SD})$ (Fig. 2a, b). His school performance was low and could not continue education after middle school. He had upper motor neuron findings at lower extremities. He had no organomegaly and lymphadenopathy. The rest of the physical examination was normal. Now, he is 25 -year-old, and using low-dose prednisolone ( $5 \mathrm{mg} /$ day), azathioprine (50 mg/day), hydroxychloroquine $(200 \mathrm{mg} /$ day $)$ and rituximab.

Skeletal radiographies showed platyspondyly without prominent metaphyseal changes (Fig. 2e, f). Cranial computerized tomography showed periventricular, frontal subcortical and basal ganglia calcification (Fig. 2c, d). Because of his claustrophobia, no MR investigation could be done. His neurologic examination was within normal limits, and he described no specific manifestation.

The physical examination of parents and two unaffected siblings (7 and 24-year old girls) was normal. Both effected siblings had systemic lupus erythematosus, spastic paraparesis, short stature, spondylo-metaphyseal dysplasia, and mild cognitive impairment. In addition, the brother had intracranial calcification. These clinical and laboratory findings were compatible with spondyloenchondrodysplasia with spasticity, cerebral calcifications, and immune dysregulation (SPENCD). To confirm the diagnosis, ACP5 gene was sequenced and c.155A >C (p.K52T) homozygous variant in ACP5 was revealed. This variant has previously been reported by Lausch et al. and Briggs et al., the latter describing two families with this variant, both of Turkish origin $[7,8]$.

\section{Search strategy}

Case-based search strategy was implemented according to a published guidance on narrative reviews [15]. We searched the PubMed/MEDLINE, Google Scholar, Web of Science and Scopus databases without any time limit, using the keywords "spondyloenchondromatosis", "type I interferonopathies", "monogenic lupus", and "pediatric-onset systemic lupus erythematosus", and found 53, 90, 148 and 98 articles, respectively. For the search "spondyloenchondrodysplasia", we excluded articles about isolated skeletal dysplasia, and included original research articles, case-based reviews, case series and case reports about spondyloenchondrodysplasia with multisystemic (neurologic and/or immunologic) 

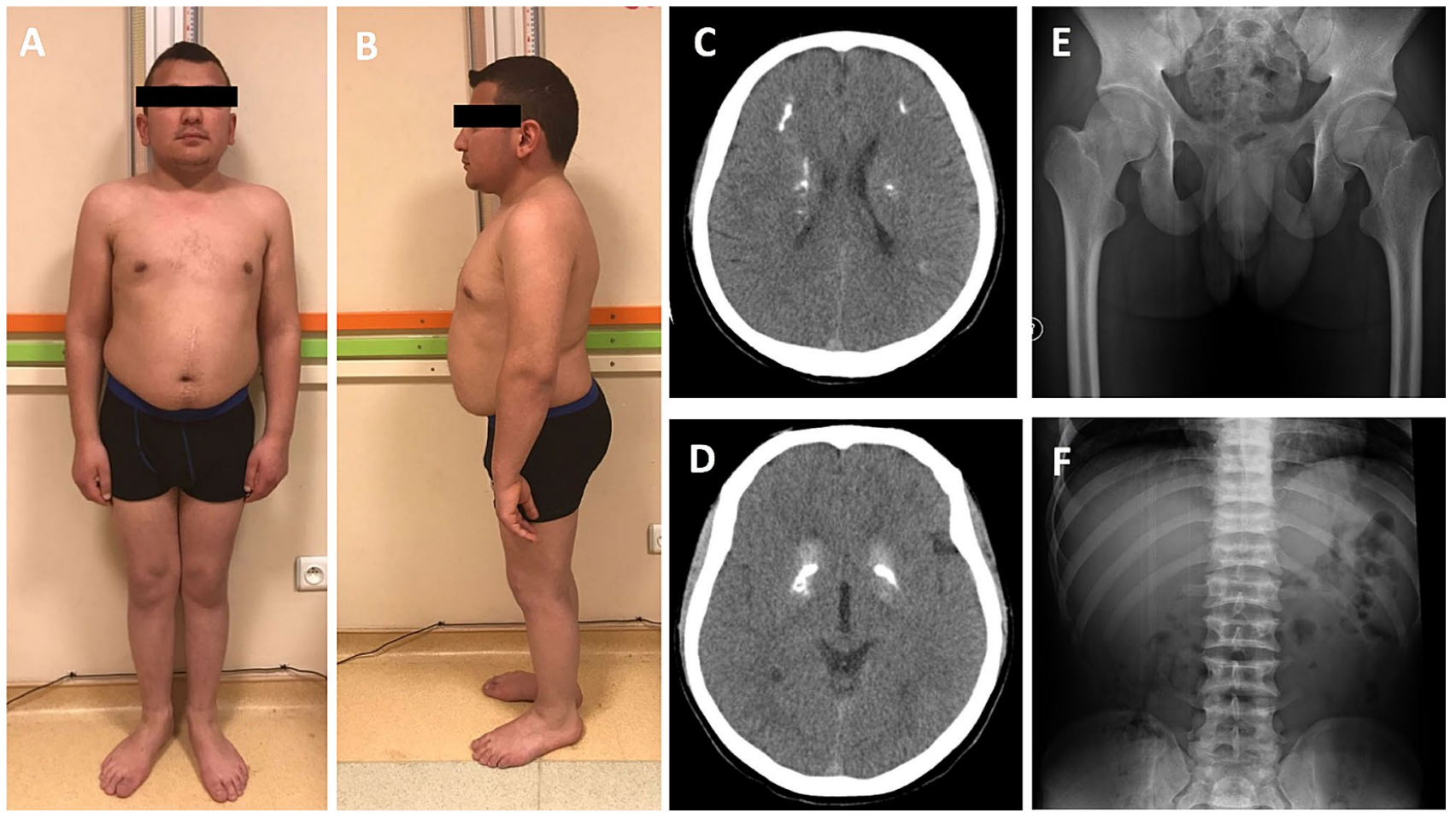

Fig. 2 Photographs of the sibling (a, b). Cranial tomography of the brain shows periventricular, subcortical and basal ganglia calcification (c, d). Normal metaphyseal view of proximal femur (e), and platyspondyly (f) of the brother

involvement, published in English, and the remaining 23 articles were reviewed. We included original research articles, case-based reviews, case reports and case series published in English for the searches "type I interferonopathies", "monogenic lupus", and "pediatric-onset systemic lupus erythematosus", and after excluded duplicates and irrelevant articles, reviewed 28, 27, and 42 articles, respectively." Informed consent was obtained from patients and their legal guards for publication.

\section{Discussion}

Type I interferonopathies are subgroups of autoinflammatory disorders and inborn errors of immunology, including Aicardi-Goutières syndrome (type 1-7), STING-associated vasculopathy, infantile-onset, (SAVI), X-linked reticulate pigmentary disorder (XLRPD), USP 18 deficiency, chronic atypical neutrophilic dermatitis with lipodystrophy (CANDLE), Singleton-Merten syndrome, $A D A 2$ deficiency and spondyloenchondrodysplasia with immune dysregulation (SPENCD), and further phenotypes may be added to this list in the future [16-19]. Inappropriate stimulation, enhanced sensitivity or defective negative regulation of the type I interferon pathway to an endogenous nucleic acid ligand and defective regulation of protein degradation are known mechanisms of type I interferonopathy phenotype $[16,19]$. The first clinical presentation in all of these diseases are seen in childhood, even in the neonatal period. Autoimmune disorders are common in type I interferonopathies, in addition to intracranial calcification, skin vasculopathy (chilblain lesions and livedo reticularis), panniculitis, skeletal problems, interstitial lung disease, failure to thrive and neurodevelopmental problems $[16,17,19]$. SLE is the first autoimmune disease identified in relation to increased interferon levels and this finding has been supported by the frequent occurrence of SLE in many type I interferonopathies to date [20]. Although, the rate of SLE due to type I interferonopathies is relatively low in all SLE cases, type I interferonopathies should be screened in pediatric-onset cases accompanied by multisystemic clinical findings described above.

SLE is thought to be caused by adaptive immune dysregulation because of the presence of high-titer autoantibodies, such as ANA and anti-dsDNA, and auto-reactive lymphocytes in affected tissues [21]. However, with the definition of Aicardi-Goutières syndrome and other type I interferonopathies, it was understood that SLE may also occur as an innate immune system disorder. It has been reported that nearly half of the SPENCD cases fulfill the criteria for SLE $[7,8,13]$. SPENCD is caused by loss of tartrate-resistant acid phosphatase (TRAP) activity, due to homozygous mutations in $A C P 5$, playing a role in increased IFN $\alpha$ production by 
plasmacytoid dendritic cells (PDCs), which is one of the main components of innate immune system. Deficient TRAP activity leads to increased phosphorylation of osteopontin (OPN), and followed by Toll-like receptor 9 (TLR-9) stimulation, increased nuclear translocation of IRF7 and NFKB, and increased expression of IFNA, interferon stimulated genes (ISGs), IL6 and TNF levels. Heterozygous missense variants of ACP5 have also been shown to significantly increase susceptibility to SLE compared to controls [13]. These findings suggest that TRAP deficiency is the main factor for susceptibility to SLE, and may be a therapeutic target in SPENCD [22]. TRAP deficiency may activate both innate and adaptive immune responses $[23,24]$. Absence of high-titer autoantibodies in early-phase of the disease in SPENCD cases, as in our case, shows that adaptive immune system was probably affected later in the disease. Defective Th1 response in TRAP knockout mice can be considered as an evidence for adaptive immune system affection [25]. However, the explicit mechanism between TRAP deficiency and susceptibility to SLE, and shared key abnormalities of innate and adaptive immune systems are not so clear.

Gender, age, race, environmental factors, epigenetic mechanisms and genetic background play a role in the pathogenesis of SLE [26]. Genetic factors become more evident in familial and early-onset $(<5$ years) SLE cases. Several monogenic causes of SLE have been described to date, having functions in complement pathway, nucleic acid repair, degradation/editing and sensing pathways, the type I interferon pathway and B cell development [27]. Mostly, the genetic etiology of monogenic SLE cases remains undetermined, according to phenotypic features and basic laboratory tests. Recently, whole exome sequencing was performed in small samples of early-onset and/or familial SLE cases, and rare causal monogenic variants associated with SLE and SLE-like phenotype identified with high penetrance [28-30]. SPENCD cases have unique findings such as short stature, platyspondyly, metaphyseal lesions, spastic paraparesis, and intracranial calcification, and may be screened easily by evaluating vertebral and long bone radiographs. However, delay in diagnosing SPENCD is frequent. We suggest to consider the spondylometaphyseal dysplasia and neurologic involvement during the evaluation of familial and/or early-onset SLE cases among other causes before detailed genetic tests.

Presentation with severe acute illness and major organ involvement are more frequent in pediatric SLE compared to adult counterparts [31-33]. Development of severe macrophage activation syndrome in the proband seems to be in accordance with these data. Renal, cutaneous and hematologic involvement were frequently reported in SPENCD cases, as in classical SLE [12,34-36]. To the best of our knowledge, there is not a case with central nervous system involvement in the literature. The frequency of renal involvement in patients with SPENCD (ranging from 67 to $82 \%$ ), is similar to those in children with multifactorial SLE [36,37]. Diffuse proliferative glomerulonephritis (class IV) is the most common form of lupus nephritis (LN) [38]. The proband had no renal involvement, but his brother had class II LN. Vitiligo, heliotrope rash, malar rash, vasculitic rash, painful vesicular lesions, oral and genital ulcers are commonly reported mucocutaneous manifestations in children with SPENCD [6-8,34-36,39]. Similarly, SLE-like mucocutaneous rashes have been developed in both of our siblings. Patients with SPENCD may present with hematologic manifestations including thrombocytopenia, anemia, autoimmune hemolytic anemia and pancytopenia, all of which are also seen in non-mendelian lupus. Refractory leukopenia/lymphopenia of the proband was the most striking hematologic finding, but there was no hematologic involvement in the brother. Arthralgia and arthritis have been reported in about two-thirds of cases in children with SPENCD [36]. Aside from the polyarthralgia episodes in the proband had, none of the siblings developed arthritis. Since there has been no approved drug in management of patients with SPENCD, these patients have been commonly treated according to the treatment guidelines of classical SLE. Among these immunosuppressive drugs, corticosteroids and hydroxychloroquine are the most commonly used agents to control disease activity in children with SPENCD [6-8,34-36,39]. Both of our patients required long-term systemic oral corticosteroids, as well as IV high dose methylprednisolone in severe episodes. Acute abdominal pain and vomiting episodes could be controlled only after rituximab in the brother. Now, both patients use azathioprine and hydroxychloroquine as maintenance therapy, and additionally brother uses small dosage oral prednisolone, and disease activity could be suppressed for 6 years, and 4 years, respectively. Targeted therapies to inhibit unabated type I IFN signaling might be a therapeutic alternative, but today a biological agent specific for SPENCD has not been described.

Also, different age of onset of clinical findings in both siblings ( 2 in female, 19 in male sibling) further supports the issue of gender bias in SLE. Very early onset of clinical findings in female sibling suggests that epigenetic and other factors may contribute to this bias without role of sex hormones.

In conclusion, our cases presented with short stature, platyspondyly, metaphyseal changes, spastic paraparesis, mild intellectual disability, and juvenile-onset SLE. Wide variety of manifestations which have been assessed by different experts and clinics without a team approach, possibly resulted with a delay in diagnosis. The diagnosis of earlyonset SLE in the proband and development of a lupus-like phenotype in brother supported the possibility of a genetic etiology. Since there are numerous genes causing monogenic form of lupus, skeletal dysplasia should be particularly 
suggestive of SPENCD in a patient with early-onset SLElike features. The patients with SPENCD generally treated in a way similar to the SLE. These cases show the importance of multisystemic evaluation in the diagnosis of SPENCD and the necessity of investigating the monogenic etiology in early-onset or familial SLE cases.

Acknowledgement We thank Dr. Yanick J. Crow for kindly analyzing the ACP5 gene sequencing.

Author contributions After interpretation of the clinical and molecular data by all of the authors (BK, ZE, SS, MG, ASG, KO, AC, AA, MI, AG, OK), BK, AG and OK conceptualized and designed the study. BK wrote the initial manuscript. All authors critically revised the manuscript at all stages of its production, final approval of manuscript, and review of literature.

\section{Compliance with ethical standards}

Conflict of interest All authors have read the manuscript and agreed with its content and stated that there is no conflict of interest.

Ethical approval All procedures performed in studies involving human participants were in accordance with the ethical standards of the institutional and/or national research committee and with the 1964 Helsinki Declaration and its later amendments or comparable ethical standards.

Informed consent Informed consent was obtained from presented cases and/or their parents.

\section{References}

1. Schorr S, Legum C, Ochshorn M (1976) Spondyloenchondrodysplasia. Radiology 118:133-139. https://doi.org/10.1148/118.1.133

2. Menger H, Kruse K, Spranger J (1989) Spondyloenchondrodysplasia. J Med Genet 26:93-99. https://doi.org/10.1136/jmg.26.2.93

3. Frydman M, Bar-Ziv J, Preminger-Shapiro R, Brezner A, Brand $\mathrm{N}$, Ben-Ami T et al (1990) Possible heterogeneity in spondyloenchondrodysplasia: quadriparesis, basal ganglia calcifications, and chondrocyte inclusions. Am J Med Genet 36:279-284. https://doi. org/10.1002/ajmg.1320360306

4. Tüysüz B, Arapoglu M, Üngür S (2004) Spondyloenchondrodysplasia: clinical variability in three cases. Am J Med Genet A 128A:185-189. https://doi.org/10.1002/ajmg.a.30078

5. Roifman C, Melamed I (2003) A novel syndrome of combined immunodeficiency, autoimmunity and spondylometaphyseal dysplasia. Clin Genet 63:522-529. https://doi.org/10.103 4/j.1399-0004.2003.00033.x

6. Renella R, Schaefer E, LeMerrer M, Alanay Y, Kandemir N, Eich $\mathrm{G}$ et al (2006) Spondyloenchondrodysplasia with spasticity, cerebral calcifications, and immune dysregulation: clinical and radiographic delineation of a pleiotropic disorder. Am J Med Genet A 140A:541-550. https://doi.org/10.1002/ajmg.a.31081

7. Briggs TA, Rice GI, Daly S, Urquhart J, Gornall H, Bader-Meunier B et al (2011) Tartrate-resistant acid phosphatase deficiency causes a bone dysplasia with autoimmunity and a type I interferon expression signature. Nat Genet 43:127-131. https://doi. org/10.1038/ng.748

8. Lausch E, Janecke A, Bros M, Trojandt S, Alanay Y, De Laet $\mathrm{C}$ et al (2011) Genetic deficiency of tartrate-resistant acid phosphatase associated with skeletal dysplasia, cerebral calcifications and autoimmunity. Nat Genet 43:132-137. https ://doi.org/10.1038/ng.749

9. Wong CK, Lit LCW, Tam LS, Li EK, Lam CW (2005) Elevation of plasma osteopontin concentration is correlated with disease activity in patients with systemic lupus erythematosus. Rheumatology 44:602-606. https://doi.org/10.1093/rheumatology/ keh558

10. D'Alfonso S, Barizzone N, Giordano M, Chiocchetti A, Magnani C, Castelli L et al (2005) Two single-nucleotide polymorphisms in the $5^{\prime}$ and $3^{\prime}$ ends of the osteopontin gene contribute to susceptibility to systemic lupus erythematosus. Arthritis Rheum 52:539-547. https://doi.org/10.1002/art.20808

11. Crow YJ (2011) Type I interferonopathies: a novel set of inborn errors of immunity. Ann N Y Acad Sci 1238:91-98. https://doi. org/10.1111/j.1749-6632.2011.06220.x

12. Briggs TA, Rice GI, Adib N, Ades L, Barete S, Baskar K et al (2016) Spondyloenchondrodysplasia due to mutations in ACP5: a comprehensive survey. J Clin Immunol 36:220-234. https:// doi.org/10.1007/s10875-016-0252-y

13. An J, Briggs TA, Dumax-Vorzet A, Alarcón-Riquelme ME, Belot A, Beresford M et al (2017) Tartrate-resistant acid phosphatase deficiency in the predisposition to systemic lupus erythematosus. Arthritis Rheumatol 69:131-142. https://doi. org/10.1002/art.39810

14. Demirkaya E, Sahin S, Romano M, Zhou Q, Aksentijevich I (2020) New horizons in the genetic etiology of systemic lupus erythematosus and lupus-like disease: monogenic lupus and beyond. J Clin Med 9:712. https://doi.org/10.3390/jcm9030712

15. Gasparyan AY, Ayvazyan L, Blackmore H, Kitas GD (2011) Writing a narrative biomedical review: considerations for authors, peer reviewers, and editors. Rheumatol Int 31:14091417. https://doi.org/10.1007/s00296-011-1999-3

16. Crow YJ (2015) Type I interferonopathies: mendelian type I interferon up-regulation. Curr Opin Immunol 32:7-12. https:// doi.org/10.1016/j.coi.2014.10.005

17. Volpi S, Picco P, Caorsi R, Candotti F, Gattorno M et al (2016) Type I interferonopathies in pediatric rheumatology. Pediatr Rheumatol 14:35. https://doi.org/10.1186/s12969-016-0094-4

18. Picard C, Bobby Gaspar H, Al-Herz W, Bousfiha A, Casanova JL, Chatila $\mathrm{T}$ et al (2018) International union of immunological societies: 2017 primary immunodeficiency diseases committee report on inborn errors of immunity. J Clin Immunol 38:96-128. https://doi.org/10.1007/s10875-017-0464-9

19. Yu Z-X, Song H-M (2020) Toward a better understanding of type I interferonopathies: a brief summary, update and beyond. World J Pediatr 16:44-51. https://doi.org/10.1007/s12519-01900273-Z

20. Hooks JJ, Moutsopoulos HM, Geis SA, Stahk SI, Decker JL, Notkins AL et al (1979) Immune Interferon in the circulation of patients with autoimmune disease. N Engl J Med 301:5-8. https ://doi.org/10.1056/NEJM197907053010102

21. de Jesus A, Goldbach-Mansky R (2016) Genetically defined autoinflammatory diseases. Oral Dis 22:591-604. https://doi. org/10.1111/odi.12448

22. Ramesh J, Parthasarathy LK, Janckila AJ, Begum F, Murugan R, Murthy BPSS et al (2020) Characterisation of ACP5 missense mutations encoding tartrate-resistant acid phosphatase associated with spondyloenchondrodysplasia. PLoS ONE 15:e230052. https ://doi.org/10.1371/journal.pone.0230052

23. Hedrich CM (2016) Shaping the spectrum-from autoinflammation to autoimmunity. Clin Immunol 165:21-28. https://doi. org/10.1016/j.clim.2016.03.002

24. Hedrich CM, Smith EMD, Beresford MW (2017) Juvenile-onset systemic lupus erythematosus (jSLE) - pathophysiological concepts and treatment options. Best Pract Res Clin Rheumatol 31:488-504. https://doi.org/10.1016/j.berh.2018.02.001 
25. Esfandiari E, Bailey M, Stokes CR, Cox TM, Evans MJ, Hayman AR et al (2006) TRACP influences Th1 pathways by affecting dendritic cell function. J Bone Miner Res 21:1367-1376. https:// doi.org/10.1359/jbmr.060611

26. Rekvig OP, Van der Vlag J (2014) The pathogenesis and diagnosis of systemic lupus erythematosus: still not resolved. Semin Immunopathol 36:301-311. https://doi.org/10.1007/s00281-014-0428-6

27. Costa-Reis P, Sullivan KE (2017) Monogenic lupus: it's all new! Curr Opin Immunol 49:87-95. https://doi.org/10.1016/j. coi.2017.10.008

28. Chung SA, Shum AK (2016) Rare variants, autoimmune disease, and arthritis. Curr Opin Rheumatol 28:346-351. https://doi. org/10.1097/BOR.0000000000000298

29. Batu ED (2018) Monogenic systemic lupus erythematosus: insights in pathophysiology. Rheumatol Int 38:1763-1775. https ://doi.org/10.1007/s00296-018-4048-7

30. Batlle-Masó L, Mensa-Vilaró A, Solís-Moruno M, MarquèsBonet T, Arostegui JI, Casals F et al (2020) Genetic diagnosis of autoinflammatory disease patients using clinical exome sequencing. Eur J Med Genet 63:103920. https://doi.org/10.1016/j. ejmg.2020.103920

31. Mina R, Brunner HI (2010) Pediatric lupus—are there differences in presentation, genetics, response to therapy, and damage accrual compared with adult lupus? Rheum Dis Clin North Am. https:// doi.org/10.1186/ar3530

32. Artim-Esen B, Şahin S, Çene E, Şahinkaya Y, Barut K, Adrovic A et al (2017) Comparison of disease characteristics, organ damage, and survival in patients with juvenile-onset and adult-onset systemic lupus erythematosus in a combined cohort from 2 tertiary centers in Turkey. J Rheumatol 44:619-625. https://doi. org/10.3899/jrheum. 160340

33. Sahin S, Adrovic A, Barut K, Canpolat N, Ozluk Y, Kilicaslan I et al (2018) Juvenile systemic lupus erythematosus in Turkey: demographic, clinical and laboratory features with disease activity and outcome. Lupus 27:514-519. https://doi.org/10.1177/09612 03317747717

34. Navarro V, Scott C, Briggs TA, Barete S, Frances C, Lebon P et al (2008) Two further cases of spondyloenchondrodysplasia (SPENCD) with immune dysregulation. Am J Med Genet A. https ://doi.org/10.1002/ajmg.a.32518

35. Girschick H, Wolf C, Morbach H, Hertzberg C, Kirsch MAL et al (2015) Severe immune dysregulation with neurological impairment and minor bone changes in a child with spondyloenchondrodysplasia due to two novel mutations in the ACP5 gene. Pediatr Rheumatol. https://doi.org/10.1186/s12969-015-0035-7

36. Bilginer Y, Düzova A, Topaloğlu R, Batu ED, Boduroğlu K, Güçer Ş et al (2016) Three cases of spondyloenchondrodysplasia (SPENCD) with systemic lupus erythematosus: a case series and review of the literature. Lupus 25:760-765. https://doi. org/10.1177/0961203316629000

37. Barron KS, Silverman ED, Gonzales J, Reveille JD (1993) Clinical, serologic, and immunogenetic studies in childhood-onset systemic lupus erythematosus. Arthritis Rheum 36:348-354. https:// doi.org/10.1002/art.1780360310

38. Brunner HI, Gladman DD, Ibanez D, Urowitz MD, Silverman ED (2008) Difference in disease features between childhood-onset and adult-onset systemic lupus erythematosus. Arthritis Rheum 58:556-562. https://doi.org/10.1002/art.23204

39. Kulkarni ML, Baskar K, Kulkarni PM (2007) A syndrome of immuno-deficiency, autoimmunity, and spondylometaphyseal dysplasia. Am J Med Genet A 143A:69-75. https://doi.org/10.1002/ ajmg.a.31526

Publisher's Note Springer Nature remains neutral with regard to jurisdictional claims in published maps and institutional affiliations. 